\title{
Avaliação clínica e radiográfica da cifoplastia no tratamento das fraturas vertebrais por osteoporose
}

Clinical and radiological evaluation of kyphoplasty in the treatment of osteoporotic spinal fractures

\author{
Evaluación clínica y radiográfica de la cifoplastia en el tratamiento \\ de las fracturas vertebrales por osteoporosis
}

\author{
Fábio Peres de Mendonça' \\ Sérgio Daher ${ }^{2}$ \\ Murilo Tavares Daher ${ }^{3}$ \\ André Luiz Passos Cardoso 4 \\ Newton Antônio Tristão ${ }^{5}$ \\ Wilson Eloy Pimenta Júnior ${ }^{5}$ \\ Ricardo Tavares Daher ${ }^{6}$
}

\section{RESUMO}

Objetivo: analisar os resultados clínicos, radiológico e possíveis complicações de pacientes submetidos à cifoplastia no tratamento das fraturas por osteoporose dolorosas que não respondem ao tratamento conservador. Métodos: foram avaliados 24 pacientes com fraturas osteoporóticas tratados através da cifoplastia. Destes, $19(76 \%)$ eram do sexo feminino e $5(24 \%)$ do masculino. A média de idade foi de 71,3 anos. A média de seguimento foi de 19 meses, variando de 3 a 29 meses. Os pacientes foram avaliados em relação à dor por meio da escala visual analógica (EVA) no pré-operatório e no último dia de seguimento. Também foi realizada uma análise radiográfica na qual se com-

\begin{abstract}
Objective: to evaluate clinical and radiological results and complications of patients undergoing kyphoplasty in the treatment of painful osteoporotic fractures. Methods: twenty-four patients with painful osteoporotic fractures were submitted do Kyphoplasty. Out of these, 19 (74\%) were female and 5 (24\%) were male. Mean age was of 71.3 years. The average follow-up (FU) was of 19 months (3 to 29). The pain was analyzed through visual analogic scale (VAS) in the pre-operative and the last follow-up visit. Radiographic analyses were made in the pre$o p$ and the last $F U$ visit using the amount of improvement in anterior vertebral body height (A), medium
\end{abstract}

\section{RESUMEN}

Objetivo: analizar los resultados clinicos, radiológicos y posibles complicaciones de pacientes sometidos a la cifoplastia en el tratamiento de las fracturas por osteoporosis dolorosas que no responden al tratamiento conservador. Métodos: fueron evaluados 24 pacientes con fracturas osteoporóticas tratados por medio de la cifoplastia. De éstos, 19 (el 76\%) fueron del sexo femenino y cinco (el 24\%) del masculino. La edad promedio fue de 71.3 años. El promedio de seguimiento fue de 19 meses, variando de 3 a 29 meses. Los pacientes fueron evaluados en relación al dolor por medio de la escala visual analógica (EVA) en el pre operatorio y en el último día de seguimiento. También fue realizado un análisis radiográfico en el cual se

\footnotetext{
Trabalho realizado no Departamento Ortopedia e Traumatologia da Faculdade de Medicina da Universidade Federal de Goiás - UFG - e no Hospital de Acidentados - Goiânia (GO), Brasil.

'Estagiário do Curso de Treinamento Avançado em Cirurgia da Coluna Vertebral da Universidade Federal de Goiás - UFG - Goiânia (GO), Brasil. ${ }^{2}$ Chefe do Serviço de Cirurgia da Coluna Vertebral e Professor Assistente do Departamento de Ortopedia e Traumatologia da Faculdade de Medicina da Universidade Federal de Goiás - UFG - Goiânia (GO), Brasil.

${ }^{3}$ Professor Auxiliar do Departamento de Ortopedia e Traumatologia Faculdade de Medicina da Universidade Federal de Goiás - UFG - Goiânia (GO), Brasil. ${ }^{4}$ Mestre em Ciências da Saúde e Médico Assistente do Serviço de Cirurgia da Coluna Vertebral do Departamento de Ortopedia e Traumatologia - UFG - Goiânia (GO), Brasil.

${ }^{5}$ Médico Assistente do Serviço de Cirurgia da Coluna Vertebral do Departamento de Ortopedia e Traumatologia da Universidade Federal de Goiás - UFG Goiânia (GO), Brasil.

${ }^{6}$ Acadêmico do Curso de Medicina da Universidade Federal de Goiás - UFG - Goiânia (GO), Brasil.

Não há conflitos de interesse.
} 
parou o ganho de altura do muro anterior (A), terço médio do corpo vertebral (M), muro posterior (P) e ganho de cifose local (C). Resultados: foram analisados 24 pacientes com 34 fraturas. Destes, $15(62,5 \%)$ apresentavam fratura única e $9(37,5 \%)$ apresentavam fraturas múltiplas. Dessas fraturas, $20(58,8 \%)$ eram na coluna torácica e $14(42,2 \%)$ lombares. A média do EVA no pré-operatório era de 9,3, passando para 3,2 no último seguimento (melhora de 6,1 pontos). $\mathrm{O}$ ganho médio de altura do corpo vertebral foi de $0,73 \mathrm{~mm}$ na porção anterior, $1,3 \mathrm{~mm}$ na porção média $\mathrm{e}$ $0,5 \mathrm{~mm}$ na porção posterior. A melhora da cifose foi, em média, de $1,32^{\circ}-$ de $11,06^{\circ}$ no pré para $12,4^{\circ}$ no pós-operatório. Em relação a complicações, houve um caso de extravasamento do cimento para o espaço discal superior, assintomático e um caso de fratura do nível adjacente tratado com nova cifoplastia. Conclusão: a cifoplastia se mostrou uma técnica cirúrgica segura e efetiva para o tratamento da dor. Não foi observado ganho significativo da altura vertebral e da cifose vertebral.

\section{DESCRITORES: Fraturas da} coluna vertebral/radiografia; Vertebroplastia; Resultado de tratamento vertebral body height $(M)$, posterior vertebral body height $(P)$, and Cobb angle (Kyphosis) in the region of the fracture. Results: 24 patients with 34 fractures were analyzed. Out of the 24 patients, 15 (62.5\%) presented with only one fracture and 9 (37.5\%) with multiple fractures. Out of these 34 fractures, 20 (58.8\%) were in the thoracic spine and $14(42.2 \%)$ in the lumbar spine. VAS average score in the pre-op was 9.3, and in the last FU it was 3.2 (improvement of 6.1). Median improvement in the anterior height was of $0.73 \mathrm{~mm}$, in the median part of $1.3 \mathrm{~mm}$, and in the posterior part of the vertebral body, $0.5 \mathrm{~mm}$. Local kyphosis improved $1.32^{\circ}$ (from $11,06^{\circ}$ to $12,4^{\circ}$ ). Two complications were observed: one case of migration of the cement to the disc and one fracture of the adjacent level. Conclusion: in this series, kyphoplasty proved to be a safe and effective technique, with good improvement of pain in the treatment of osteoporotic fractures. No significant improvement in vertebral height and kyphosis was observed.

\author{
KEYWORDS: Spinal fractures/ \\ radiography; Vertebroplasty; \\ Treatment outcome
}

comparó la ganancia de altura del muro anterior (A), tercio medio del cuerpo vertebral (M), muro posterior (P) y la ganancia de la cifosis local (C). Resultados: fueron analizados 24 pacientes con 34 fracturas. De éstos 15 (el 62.5\%) presentaban fractura única y 9 (el 37.5\%), fracturas múltiples. De estas fracturas, 20 (el 58.8\%) fueron en la columna torácica y 14 (el 42.2\%), lumbares. El promedio del EVA en el pre operatorio fue de 9.3, pasando para 3.2 en el último seguimiento (mejora de 6.1 puntos). La ganancia en promedio de la altura del cuerpo vertebral fue de $0.73 \mathrm{~mm}$ en la porción anterior, $1.3 \mathrm{~mm}$ en la porción media y $0.5 \mathrm{~mm}$ en la porción posterior. La mejoría de la cifosis fue en promedio de $1.32^{\circ}$, de $11.06^{\circ}$ en el pre operatorio para $12.4^{\circ}$ en el post operatorio. Como complicaciones, hubo un caso de extravasamiento del cemento para el espacio discal superior y asintomático, y un caso de fractura del nivel adyacente tratado con nueva cifoplastia. Conclusión: la cifoplastia se mostró una técnica quirúrgica segura y efectiva para el tratamiento del dolor. No fue observada una ganancia significativa de la altura vertebral y de la cifosis vertebral.

\section{DESCRIPTORES: Fracturas} de la columna vertebral/ radiografia; Vertebroplastía; Resultado del tratamiento

\section{INTRODUÇÃO}

As fraturas vertebrais osteoporóticas são prevalentes na população idosa, e sua incidência vem aumentando com o envelhecimento da população. Podem ser assintomáticas, mas, muitas vezes, causam sintomas como dor e deformidade, estando relacionadas a uma piora da qualidade de vida e aumento da mortalidade nessa população ${ }^{1,2}$.

Usualmente, essas fraturas respondem bem ao tratamento conservador por meio de repouso, analgésicos e coletes. No entanto, o repouso acelera a perda de massa óssea, agravando a osteoporose, e a medicação pode trazer inúmeros efeitos adversos, principalmente na população idosa. Além disso, os coletes muitas vezes não são bem tolerados por esse grupo de pacientes ${ }^{2,3}$.

O tratamento clínico da osteoporose consiste na tentativa de diminuir a perda óssea por meio do uso de bifosfonados, análogos de paratormônio, calcitonina e reposição hormonal em mulheres pós-menopausa. Porém, em fraturas agudas, esses tratamentos não promovem melhora da dor, exceto a calcitonina que parece ter ação analgésica ${ }^{4}$.

Para aqueles casos refratários ao tratamento conservador, uma opção é a cimentação vertebral. A vertebroplastia foi descrita na França por Gilbert e Deramond, inicialmente para o tratamento de um hemangioma de $\mathrm{C}^{5}$, e posteriormente usada no tratamento de outras lesões vertebrais (osteoporose e lesões osteolíticas) $^{6-8}$.

A cifoplastia é uma evolução da técnica da vertebroplastia por possuir uma etapa inicial que consiste em insuflar um balão dentro do corpo vertebral, criando uma cavidade para o preenchimento do cimento9. Isso proporcionaria maior segurança, pois o cimento é injetado sob 
baixa pressão e permite redução da fratura e melhora da deformidade no plano sagital ${ }^{2}$.

O objetivo deste estudo foi analisar os pacientes submetidos à cifoplastia quanto à melhora da dor, ao ganho de altura vertebral e a possíveis complicações.

\section{MÉTODOS}

O estudo foi aprovado pelo Comitê de Ética local. Foram incluídos 24 pacientes com fraturas osteoporóticas da coluna vertebral, tratados através da cifoplastia no período de Junho de 2007 a Dezembro de 2009. Destes, 19 (76\%) eram do sexo feminino e $5(24 \%)$ do sexo masculino. A média de idade foi de 71,3 anos, variando de 66 a 82. O seguimento médio dos pacientes foi de 19 meses, variando de 3 a 29 meses.

Os critérios de inclusão foram: fratura por osteoporose dolorosa, falha do tratamento conservador por, no mínimo, quatro semanas, dor localizada na altura da fratura e ressonância magnética (RM) mostrando hipersinal nas imagens ponderadas em T2 com saturação de gordura.

Foram excluídos os casos com fraturas patológicas por tumores vertebrais e fraturas com acometimento importante do muro posterior.

A análise funcional foi realizada pré-operatoriamente e no último dia de seguimento por meio da escala visual analógica de dor.

A avaliação radiográfica foi realizada no pré-operatório e no último dia de seguimento por meio dos seguintes parâmetros:

- altura anterior (A): altura do muro anterior do corpo vertebral medido na radiografia em perfil (Figura 1);

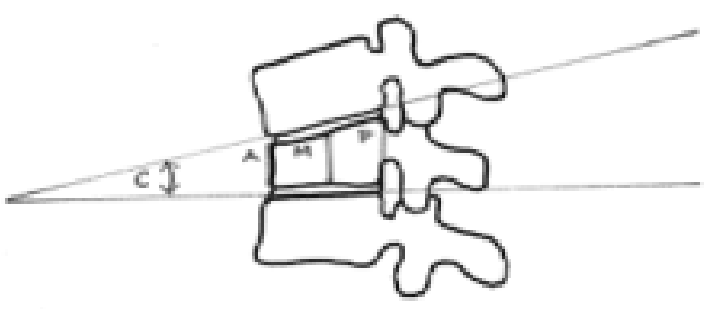

Figura 1

Parâmetros radiográficos analisados. A - altura anterior, $M-$ altura média, $P$ - altura posterior.

- altura média (M): altura no ponto médio do platô superior e inferior medido na radiografia de perfil (Figura 1);

- altura posterior (P): altura do muro posterior do corpo vertebral também na radiografia em perfil (Figura 1);

- cifose vertebral (C): ângulo de Cobb medido através do platô inferior da vértebra superior e do platô superior da vértebra inferior à fraturada (Figura 1). Nos casos de fraturas adjacentes foi feita a medida utilizando-se as vértebras adjacentes íntegras.

Foram analisadas as principais complicações: déficit neurológico, extravasamento de cimento (por meio da análise das radiografias no pós-operatório) e fraturas dos níveis adjacentes até o último dia de seguimento.

\section{Técnica cirúrgica}

Todos os pacientes foram operados em decúbito ventral em mesa radiotransparente sobre coxins sob sedação e anestesia local.

A abordagem transpedicular (Figura 2A) foi indicada nas fraturas abaixo de T10. Acima desse nível foi utilizada a abordagem extrapedicular (Figura 2B). A abordagem bilateral foi realizada em todos os casos objetivando-se a restauração da altura do corpo vertebral ao inflar o balão.

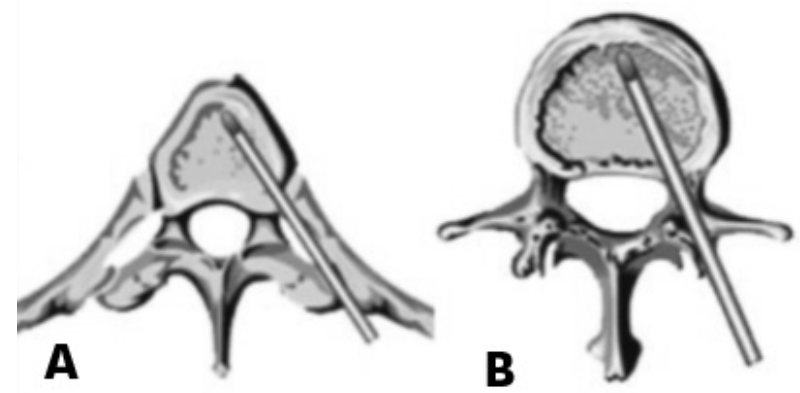

Figura 2

Abordagem extrapedicular e transpedicular.

Após a confirmação do posicionamento do balão no corpo vertebral, estes eram insuflados com controle de volume e pressão, sendo os limites a capacidade volumétrica do balão, o limite de pressão do balão, redução da fratura e risco de violação da cortical óssea. Ao fim da insuflação é obtido o volume de cimento a ser injetado em cada cavidade.

A introdução do cimento ósseo é realizada após a retirada dos balões, sendo que a consistência ideal é próxima à de uma "pasta de dente", nunca sob a forma liquefeita, devido ao maior risco de extravasamento (Figura 3).

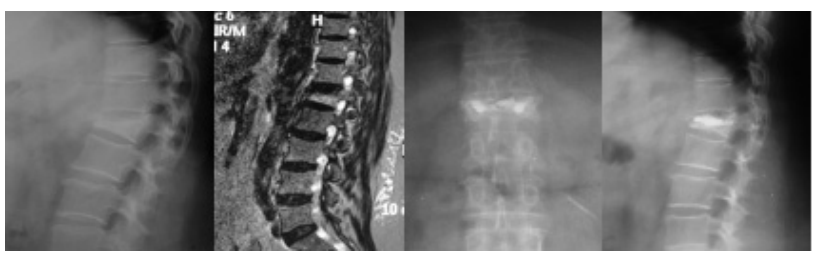

Figura 3

Caso clínico. Raio X mostrando fratura de $L 1$ que não respondeu ao tratamento clínico. RM mostrando imagem sagital ponderada em T2 com supressão de gordura (STIR) com hipersinal em T1. Raio $X$ pós-operatório do último seguimento mostrando boa cimentação da vértebra.

\section{RESULTADOS}

Os dados dos pacientes estão apresentados na Tabela 1. Foram avaliados 24 pacientes com 34 fraturas tratadas. Destes, $15(62,5 \%)$ apresentavam fratura em um único nível e $9(37,5 \%)$ em mais de um nível. Das 34 fraturas submetidas ao procedimento, $20(58,8 \%)$ eram no nível torácico e $14(41,2 \%)$ lombares. 
TABELA 1 - Dados clínicos, radiológicos e complicações

\begin{tabular}{|c|c|c|c|c|c|c|c|c|c|c|}
\hline & \multirow[t]{2}{*}{ Paciente } & \multirow[t]{2}{*}{ Idade } & \multirow[t]{2}{*}{ Sexo } & \multirow[t]{2}{*}{$N^{\circ}$ fraturas } & \multirow{2}{*}{$\begin{array}{l}\text { Tempo de } \\
\text { fratura } \\
\text { (Semanas) }\end{array}$} & \multicolumn{3}{|c|}{$\begin{array}{l}\text { Ganho de altura por porção } \\
\text { do corpo em milímetros (mm) }\end{array}$} & \multirow{2}{*}{$\begin{array}{l}\text { Ganho } \\
\text { angular }\end{array}$} & \multirow[t]{2}{*}{ Complicação } \\
\hline & & & & & & (A) & $(M)$ & (P) & & \\
\hline 1 & MRA & 69 & $M$ & T12 & 5 & (1) & (1) & (0) & $3^{\circ}$ & \\
\hline 2 & $\mathrm{CBL}$ & 66 & $M$ & T12; L1 & 4 & $(0 ; 3)$ & $(0 ; 3)$ & $(1 ; 0)$ & $0^{\circ} ; 4^{\circ}$ & \\
\hline 3 & MCSL & 82 & $M$ & T9; L2 & 5 & $(0 ; 1)$ & $(0 ; 1)$ & $(0 ; 0)$ & $0^{\circ} ; 3^{\circ}$ & \\
\hline 4 & CSG & 68 & $\mathrm{H}$ & $\mathrm{T} 11 ; \mathrm{T} 12$ & 4 & $(1 ; 0)$ & $(2 ; 0)$ & $(0 ; 0)$ & $3^{\circ} ; 0^{\circ}$ & $\begin{array}{l}\text { Fratura } \\
\text { adjacente no } \\
\text { sexto mês }\end{array}$ \\
\hline 5 & MGM & 70 & $M$ & T12; L1 & 5 & $(0 ; 0)$ & $(0 ; 3)$ & $(0 ; 0)$ & $0^{\circ} ; 0^{\circ}$ & \\
\hline 6 & $\| \mathrm{ILD}$ & 67 & $M$ & T9 & 4 & (2) & (4) & (2) & 0 & \\
\hline 7 & IAE & 66 & $M$ & L1 & 5 & (5) & (5) & (2) & 5 & \\
\hline 8 & $\mathrm{IBM}$ & 75 & $M$ & L2 & 12 & (0) & (0) & (0) & 0 & \\
\hline 9 & MJDS & 68 & $M$ & T12; L3 & 5 & $(2 ; 0)$ & $(3 ; 0)$ & $(2 ; 0)$ & $2^{\circ} ; 0^{\circ}$ & \\
\hline 10 & GC & 71 & $\mathrm{H}$ & T11 & 5 & (1) & $(2)$ & (1) & 3 & \\
\hline 11 & MCF & 75 & $M$ & T11; L1 & 5 & (0) & (1) & (0) & 0 & \\
\hline 12 & EFL & 72 & M & $\mathrm{T} 11$ & 5 & (0) & (1) & (0) & 0 & \\
\hline 13 & MMS & 67 & $M$ & $\lfloor 4$ & 4 & (0) & (2) & (1) & 2 & \\
\hline 14 & CVB & 68 & M & $\begin{array}{l}\text { T8; T10; } \\
\text { Tा1 }\end{array}$ & 4 & $(0 ; 0 ; 1)$ & $(0 ; 0 ; 3)$ & $(0 ; 0 ; 1)$ & $0^{\circ} ; 0^{\circ} ; 2^{\circ}$ & \\
\hline 15 & $\mathrm{OHS}$ & 72 & $\mathrm{H}$ & L1 & 4 & (0) & (0) & (0) & 0 & \\
\hline 16 & MSP & 66 & $M$ & $\mathrm{~T} 12$ & 5 & (1) & (2) & (2) & 2 & \\
\hline 17 & ARP & 70 & $M$ & T12 & 4 & (2) & (2) & (2) & 0 & \\
\hline 18 & MAN & 71 & $M$ & L3 & 4 & (0) & (0) & $(0)$ & 0 & \\
\hline 19 & TCC & 76 & $M$ & T11; L1 & 6 & $(0 ; 1)$ & $|0 ; 3|$ & $(0 ; 2)$ & $0^{\circ} ; 4^{\circ}$ & \\
\hline 20 & $J P$ & 76 & $\mathrm{H}$ & L4 & 5 & (0) & (0) & (0) & 0 & \\
\hline 21 & YK & 70 & M & T9; Т12 & 4 & $(1 ; 0)$ & $(2 ; 0)$ & $(1 ; 0)$ & $4^{\circ} ; 0^{\circ}$ & \\
\hline 22 & WJVR & 73 & $\mathrm{H}$ & L2 & 4 & (1) & (1) & (0) & $2^{\circ}$ & \\
\hline 23 & EFM & 76 & $M$ & $\mathrm{~T} 12$ & 6 & (1) & (1) & (0) & $2^{\circ}$ & \\
\hline 24 & CPD & 79 & $M$ & $\mathrm{LI}$ & 4 & (1) & (2) & (0) & $4^{\circ}$ & $\begin{array}{l}\text { Migração do } \\
\text { cimento }\end{array}$ \\
\hline
\end{tabular}

O tempo médio do diagnóstico da fratura ao procedimento foi de 5 semanas, variando de 4 a 12 semanas.

O ganho médio de altura no corpo vertebral observado foi de: $0,73 \mathrm{~mm}$ na porção anterior, $1,3 \mathrm{~mm}$ na porção média e $0,5 \mathrm{~mm}$ na porção posterior.

O ganho em relação à correção do ângulo de cifose vertebral em média foi de $1,32^{\circ}$, sendo que a média pré-operatória era de $11,06^{\circ}$, indo para $12,4^{\circ}$ no pós-operatório (Tabela 1 ).

Como complicação precoce não ocorreu nenhum caso de extravasamento de cimento para o canal ou déficit neurológico. Houve um caso de migração do cimento para o espaço discal superior. Como complicação tardia foi observada uma fratura no nível adjacente no sexto mês de pós-operatório. O tratamento realizado foi uma nova cifoplastia no nível adjacente no caso com nova fratura, e observação do paciente com extravasamento do cimento, pois o mesmo encontrava-se assintomático.

A avaliação pela escala visual analógica (EVA) mostrou uma melhora média de 6,1 pontos com decréscimo médio de 9,3 para 3,2 pontos (Gráfico 1 e Tabela 2).

\section{DISCUSSÃO}

O uso da cimentação vertebral no tratamento das fraturas por osteoporose tem sido uma boa opção naqueles casos

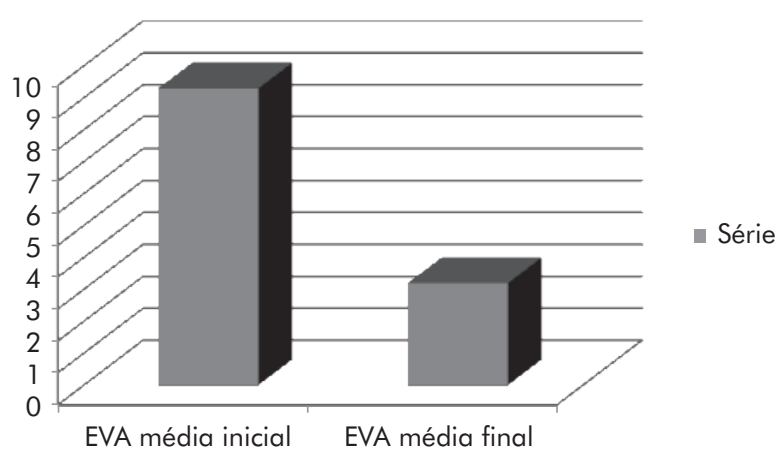

Gráfico 1

Avaliação pré e pós-operatória pela escala visual analógica (EVA)

que não respondem ao tratamento conservador. Vários estudos têm mostrado boa melhora clínica com esse método e baixos índices de complicações ${ }^{7-9}$.

O momento ideal de se indicar uma intervenção cirúrgica, mesmo que minimamente invasiva, em pacientes com fraturas osteoporóticas vertebrais, permanece incerto. Concordamos com a maioria dos autores que uma tentativa prévia do tratamento conservador deve sempre ser realizada, já que a maioria desses pacientes evolui 


\begin{tabular}{|c|c|c|c|}
\hline & Paciente & $\begin{array}{c}\text { EVA } \\
\text { Pré-operatória }\end{array}$ & $\begin{array}{c}\text { EVA } \\
\text { Pós-operatória }\end{array}$ \\
\hline 1 & MRA & 8 & 0 \\
\hline 2 & CBL & 9 & 4 \\
\hline 3 & MCSL & 10 & 2 \\
\hline 4 & CSG & 9 & 2 \\
\hline 5 & $M G M$ & 10 & 3 \\
\hline 6 & $\| \mathrm{LD}$ & 10 & 6 \\
\hline 7 & IAE & 9 & 3 \\
\hline 8 & IBM & 10 & 5 \\
\hline 9 & MIDS & 10 & 3 \\
\hline 10 & GC & 9 & 4 \\
\hline 11 & MCF & 10 & 5 \\
\hline 12 & $\mathrm{EFL}$ & 10 & 6 \\
\hline 13 & MMS & 9 & 3 \\
\hline 14 & CVB & 10 & 4 \\
\hline 15 & $\mathrm{OHS}$ & 9 & 4 \\
\hline 16 & MSP & 10 & 3 \\
\hline 17 & ARP & 10 & 3 \\
\hline 18 & MAN & 8 & 0 \\
\hline 19 & TCC & 9 & 3 \\
\hline 20 & $J P$ & 9 & 2 \\
\hline 21 & YK & 10 & 3 \\
\hline 22 & WJVR & 7 & 0 \\
\hline 23 & EFM & 9 & 5 \\
\hline 24 & CPD & 9 & 4 \\
\hline
\end{tabular}

satisfatoriamente com a melhora do quadro álgico. Em nosso estudo, consideramos que o tempo mínimo para se indicar a cifoplastia deve ser de quatro semanas de tratamento.

Em nossa casuística houve uma melhora considerável da dor após o procedimento. O mesmo foi observado por Coumans em seu trabalho prospectivo com seguimento mínimo de um ano em 188 fraturas de 78 pacientes. Nesse estudo, o índice de Owestry e a escala visual de dor demonstraram melhora no pós-operatório imediato, resultado que se manteve em todo o seguimento ${ }^{10}$.
Em nosso meio, Oliveira apresentou uma melhora na EVA de 6,8, em média, resultado semelhante ao encontrado em nosso estudo ${ }^{7}$. Também Pinto mostrou melhora consistente da dor com o procedimento ${ }^{8}$. No primeiro estudo, os autores descrevem um caso de extravasamento do cimento para dentro do canal durante a realização da vertebroplastia com déficit neurológico e, no segundo, os autores descrevem um caso de colapso da vértebra adjacente e um caso de preenchimento do plexo venoso perivertebral ${ }^{8}$. Em nosso estudo, tivemos também um caso de fratura do nível adjacente, e nenhum caso de extravasamento do cimento para dentro do canal raquiano.

O mecanismo preciso de como ocorre a melhora da dor ainda é objeto de estudo. Segundo alguns autores, a melhora se deve a uma diminuição dos micromovimentos da vértebra fraturada, associada à lesão térmica e tóxica das terminações vertebrais secundárias à aplicação do cimento $^{11,12}$.

Em relação ao ganho de altura e à melhora do ângulo de cifose com a cifoplastia, existem controvérsias sobre os ganhos serem secundários ao posicionamento na mesa cirúrgica ou à insuflação do balão ${ }^{2,13}$. Em nossa casuística, o ganho de altura e do grau de cifose, apesar de observado, não foi considerável.

Em nossa opinião, a grande vantagem da cifoplastia frente à vertebroplastia é a diminuição do risco de extravasamento de cimento ${ }^{14}$. Em nossa casuística houve apenas um caso de extravasamento para o disco adjacente. Porém, se tivéssemos utilizado a tomografia, provavelmente esse número seria consideravelmente maior.

Já o ganho de altura da vértebra parece não ser tão consistente, já que em nossa população esse ganho não foi importante. Além disso, mesmo quando ocorre uma redução da fratura, parece não haver repercussão no equilíbrio sagital do paciente ${ }^{2}$.

\section{CONCLUSÃO}

A cifoplastia se mostrou uma técnica cirúrgica segura e efetiva para o tratamento das fraturas por osteoporose dolorosas em casos que não respondem ao tratamento clínico. Não foi observado ganho expressivo da altura vertebral e melhora da cifose vertebral.

\section{REFERÊNCIAS}

1. Hall SE, Criddle RA, Comito TL, Prince RL. A case-control study of quality of life and functional impairment in women with longstanding vertebral osteoporotic fracture. Osteoporos Int. 1999;9(6):508-15.
2. Pradhan BB, Bae HW, Kropf MA, Patel VV, Delamarter RB. Kyphoplasty reduction of osteoporotic vertebral compression fractures: correction of local kyphosis versus overall sagittal alignment. Spine (Phila Pa 1976). 2006:31(4):435-41.
3. Garfin SR, Yuan HA, Reiley MA. New technologies in spine: kyphoplasty and vertebroplasty for the treatment of painful osteoporotic compression fractures. Spine (Phila $\mathrm{Pa}$ 1976).2001;26(14):1511-5.

4. Kapuściński P, Talalaj M, Borowicz J, Marcinowska-Suchowierska E, Brzozowski R. An analgesic effect of synthetic human calcitonin in patients with primary osteoporosis. Mater Med Pol. 1996;28(3):83-6. 
5. Galibert P, Deramond H, Rosat $P$, Le Gars D. Preliminary note on the treatment of vertebral angioma by percutaneous acrylic vertebroplasty. Neurochirurgie. 1987;33(2):166-8.

6. Deramond H, Depriester C, Galibert P, Le Gars D.

Percutaneous vertebroplasty with polymethylmethacrylate. Technique, indications, and results. Radiol Clin North Am. 1998;36(3):533-46.

7. Oliveira FM, Rodrigues AG, Bastos Junior JOC, Yamazoto C, Kusabara R. Resultados clínicos da vertebroplastia em pacientes com fratura da coluna vertebral por osteoporose. Coluna/ Columna. 2007;6(1):28-33.

8. Pinto R, Matos R, Neves N, Tulha JM, Cabral AT, Almeida L. A vertebroplastia no tratamento das fracturas vertebrais osteoporóticas dolorosas. Coluna/Columna . 2007:6(3):136-40.
9. Lovi A, Teli M, Ortolina A, Costa F, Fornari M, Brayda-Bruno M. Vertebroplasty and kyphoplasty: complementary tecniques for the treatment of painful osteoporotic vertebral compression fractures. Coluna/Columna . 2009;8(1):57-62.

10.Coumans JV, Reinhardt MK, Lieberman IH. Kyphoplasty for vertebral compression fractures: 1-year clinical outcomes from a prospective study. J Neurosurg. 2003;99(1 Suppl):44-50.

11.Belkoff SM, Maroney M, Fenton DC, Mathis JM. An in vitro biomechanical evaluation of bone cements used in percutaneous vertebroplasty. Bone. 1999:25(2 Suppl):23S-6S.

12.Deramond H, Wright NT, Belkoff SM. Temperature elevation caused by bone cement polymerization during vertebroplasty. Bone. 1999:25(2 Suppl):17S-21S.
13.Ledlie JT, Renfro MB. Decreases in the number and severity of morphometrically defined vertebral body deformities after kyphoplasty. Neurosurg Focus. 2005;18(3):e4.

14.Heini PF, Orler R. Kyphoplasty for treatment of osteoporotic vertebral fractures. Eur Spine J. 2004;13(3):18492.

\section{Correspondência}

Fábio Peres de Mendonça

Hospital São Mateus

Av. das Flores, 355

Bosque da Saúde

CEP 78043-172

E-mail: fabiopmendonca@bol.com.br 